\title{
Generalized Pseudopotentials for the Anisotropic Fractional Quantum Hall Effect
}

\author{
Bo Yang, ${ }^{1}$ Zi-Xiang Hu, ${ }^{2}$ Ching Hua Lee, ${ }^{3}$ and Z. Papić ${ }^{4}$ \\ ${ }^{1}$ Complex Systems Group, Institute of High Performance Computing, A*STAR, Singapore 138632, Singapore \\ ${ }^{2}$ Department of Physics, Chongqing University, Chongqing 401331, People's Republic of China \\ ${ }^{3}$ Material Science and Engineering, Institute of High Performance Computing, $A * S T A R$, Singapore 138632, Singapore \\ ${ }^{4}$ School of Physics and Astronomy, University of Leeds, Leeds LS2 9JT, United Kingdom
}

(Received 25 September 2016; published 5 April 2017)

\begin{abstract}
We generalize the notion of Haldane pseudopotentials to anisotropic fractional quantum Hall $(\mathrm{FQH})$ systems that are physically realized, e.g., in tilted magnetic field experiments or anisotropic band structures. This formalism allows us to expand any translation-invariant interaction over a complete basis, and directly reveals the intrinsic metric of incompressible FQH fluids. We show that purely anisotropic pseudopotentials give rise to new types of bound states for small particle clusters in the infinite plane, and can be used as a diagnostic of $\mathrm{FQH}$ nematic order. We also demonstrate that generalized pseudopotentials quantify the anisotropic contribution to the effective interaction potential, which can be particularly large in models of fractional Chern insulators.
\end{abstract}

DOI: 10.1103/PhysRevLett.118.146403

The fractional quantum Hall (FQH) system is host to a wide variety of topological phases of matter [1]. This complexity belies the deceivingly simple microscopic Hamiltonian containing only the effective Coulomb interaction projected to a single Landau level (LL) [2]. The understanding of different topological states was greatly facilitated by the concept of pseudopotentials (PPs) introduced by Haldane [3,4]. This formalism allows one to expand any rotation-invariant interaction over the complete basis of the PPs, which are projection operators onto two-particle states with a given value of relative angular momentum. Furthermore, a combination of a small number of PPs naturally defines parent Hamiltonians for some FQH model states, such as the Laughlin states $[5,6]$. The method has also been generalized to many-body PPs $[7,8]$, which form the parent Hamiltonians of the non-Abelian FQH states $[9,10]$. In many cases, the ground state of these model Hamiltonians is believed to be adiabatically connected to the actual ground state of the experimental system. Thus, the relatively simple (and to some degree analytically tractable) model wave functions and Hamiltonians give much insight into the nature of the experimentally realized $\mathrm{FQH}$ states.

Recently, interest in the FQH effect has been renewed due to emerging connections between topological order, geometry, and broken symmetry. An early precursor of these ideas was the realization that rotational invariance is not necessary for the FQH effect [4]. This lead to the conclusion that $\mathrm{FQH}$ states possess new "geometrical" degrees of freedom [11], uncovering a more complete description of their low-energy properties [12-14]. The notion of geometry has also inspired the construction of a more general class of Laughlin states with the nonEuclidean metric [15], which was shown to be physically relevant in situations where the band mass or dielectric tensor is anisotropic [16-18], or in the tilted magnetic field [19]. On the other hand, an intriguing coexistence of topological order with broken symmetry [20,21], leading to the nematic FQH effect, has also been proposed $[22,23]$. The nematic order is expected to arise due to spontaneous symmetry breaking, as suggested by recent numerical evidence [24] and experiment [25].

A missing ingredient for the complete microscopic description of the mentioned geometry and broken symmetry in the $\mathrm{FQH}$ effect is the formulation of PPs for systems without rotational invariance. A rotationally invariant system is one where the effective interaction $V\left(q_{x}, q_{y}\right)$, which encompasses the bare Coulomb potential and the LL form factor [2], is a function of a single metric $g$, i.e., $V\left(q_{x}, q_{y}\right)=V\left(|q|=\sqrt{g^{a b} q_{a} q_{b}}\right)$. Note that the metric $g$ does not necessarily have to be Euclidean in the lab frame, because its physics remain unchanged upon a trivial coordinate transformation back to the Euclidean metric. By contrast, if the Coulomb potential and LL form factor are characterized by different metrics, rotational symmetry is "truly" broken. Note that a finite system can break rotational invariance by its boundary conditions, but recent work [8] has shown that conventional PPs can nevertheless be defined in such cases. In contrast, here we focus on describing a thermodynamically large system without rotational invariance. Such systems are highly relevant for experiment, yet they cannot be described by the conventional PP formalism.

In this paper, we extend Haldane's approach [3] and construct a complete basis of the generalized PPs for any two-body effective interaction. An immediate benefit of the generalized PPs is that anisotropic FQH systems, such as tilted magnetic field experiments, can be studied in a 
quantitative and universal language. Moreover, the generalized PPs lead to several physical consequences for $\mathrm{FQH}$ systems. First, we show that ground states of purely anisotropic PPs feature new types of bound states with "molecular" structures. In combination with isotropic PPs, such molecular clusters might give rise to novel types of order in partially filled LLs. Second, we show that the anisotropic PPs are a natural probe of the simplest FQH nematic states [22,23], and motivate the construction of a more general class of orders that extend beyond the "quadrupolar" instability of neutral excitations of the Laughlin state. Third, we explicitly evaluate the anisotropic PPs in a model fractional Chern insulator (FCI), and show they are comparable in magnitude to the isotropic PPs. This suggests that anisotropic PPs are not only physically relevant in FCIs, but can be useful for determining the optimal FCI models for realizing various topological states. Finally, we highlight some broader connections between generalized PPs and the emergent geometry of $\mathrm{FQH}$ systems.

Generalized pseudopotentials.-Any two-body $\mathrm{FQH}$ Hamiltonian, projected to a single LL, is given by

$$
\mathcal{H}=\int \frac{d^{2} \vec{q} \ell_{B}^{2}}{(2 \pi)^{2}} V_{\vec{q}} \bar{\rho}_{\vec{q}} \bar{\rho}_{-\vec{q}},
$$

where $\bar{\rho}_{\vec{q}}=\sum_{i} e^{i q_{a} R_{i}^{a}}, a=(x, y)$ is the guiding-center density operator with momentum $\vec{q}=\left(q_{x}, q_{y}\right)$ (we assume the Einstein summation convention). The model in Eq. (1) is believed to explain most of the observed $\mathrm{FQH}$ states depending on the subtle energetics dictated by the electron filling fraction $\nu$ and the effective interaction potential $V_{\vec{q}}$. The complexity of $\mathcal{H}$ in Eq. (1) is hidden in the fact that the guiding center coordinates do not commute: $\left[R_{i}^{a}, R_{j}^{b}\right]=$ $-i \epsilon^{a b} \ell_{B}^{2} \delta_{i, j}$, where $\epsilon^{a b}$ is the antisymmetric tensor and $\ell_{B}$ is the magnetic length (we set $\ell_{B}=1$ ).

Interaction $V_{\vec{q}}$ can be written as $V_{\vec{q}}=V_{\vec{q}}^{\text {bare }}\left|F_{N}(\vec{q})\right|^{2}$, where $V_{\vec{q}}^{\text {bare }}$ is the bare Coulomb potential and $F_{N}(\vec{q})$ the form factor of the electrons in the $N$ th LL. In the simplest case of a sample with infinitesimal thickness in the perpendicular direction, $F_{N}(\vec{q})=F_{N}\left(|q|_{l}\right)$ and $V_{\vec{q}}^{\text {bare }}=V_{|q|_{c}}^{\text {bare }}$, with $|q|_{l(c)}^{2}=$ $q_{a} q_{b} g_{l(c)}^{a b}$. The LL metric $g_{l}$ parametrizes the effective mass tensor, and the Coulomb metric $g_{c}$ describes the shape of the equipotential lines around the electrons in a dielectric [11]. In the following, we first assume isotropic metrics $g_{l}=g_{c}=$ $g=\mathbb{1}_{2 \times 2}$, and return to the more general case in Eq. (6) at the end.

For two particles, the center of mass guiding-center coordinates commute with the Hamiltonian of Eq. (1). Thus, the relevant Hilbert space is spanned by two-particle eigenstates $|m\rangle$ of relative angular momentum $m$ [4]. Assuming $\Delta m \equiv\left(m^{\prime}-m\right)>0$, we obtain the following expression for the matrix element: $\left\langle m\left|\bar{\rho}_{\vec{q}} \bar{\rho}_{-\vec{q}}\right| m^{\prime}\right\rangle=\sqrt{\frac{m !}{m^{\prime} !}}\left(i \sqrt{2} \mathbf{q}^{*}\right)^{\Delta m} e^{-\frac{1}{2}|q|^{2}} L_{m}^{\Delta m}\left(|q|^{2}\right)$.

Here $m, m^{\prime}$ are necessarily even for bosons and odd for fermions, $\mathbf{q} \equiv(1 / \sqrt{2})\left(q_{x}+i q_{y}\right)$, and $L_{m}^{n}(x)$ is the generalized Laguerre polynomial. From Eq. (1) it is clear that if $V_{\vec{q}}$ is rotationally invariant with $g_{l}=g_{c}=g=\mathbb{1}_{2 \times 2}$, then $\left\langle m|\mathcal{H}| m^{\prime}\right\rangle \sim \sum_{n} c_{n} \delta_{m, n} \delta_{m^{\prime}, n}$. It is thus useful to decompose the interaction into the standard Haldane PPs $V_{m}(|q|)$ as $V_{|q|}=\sum_{m=0}^{\infty} c_{m} V_{m}(|q|)$, where $V_{m}(|q|)=e^{-\frac{1}{2}|q|^{2}} L_{m}\left(|q|^{2}\right)$. Many of the well-known FQH states are described by model Hamiltonians formed by linear combinations of select $V_{m}$.

We next focus on arbitrary anisotropic $V_{\vec{q}}$. In this case, the Haldane PPs no longer form a complete basis. We define the generalized PPs as follows:

$$
\begin{aligned}
& V_{m, n}^{+}(\vec{q})=\lambda_{n} \mathcal{N}_{m n}\left(L_{m}^{n}\left(|q|^{2}\right) e^{-\frac{1}{2}|q|^{2}} \mathbf{q}^{n}+\text { c.c. }\right), \\
& V_{m, n}^{-}(\vec{q})=-i \mathcal{N}_{m n}\left(L_{m}^{n}\left(|q|^{2}\right) e^{-\frac{1}{2}|q|^{2}} \mathbf{q}^{n}-\text { c.c. }\right),
\end{aligned}
$$

where the normalization factors are $\mathcal{N}_{m n} \equiv$ $\sqrt{2^{n-1} m ! /(\pi(m+n) !)}$, and $\lambda_{n}=1 / \sqrt{2}$ for $n=0$ or $\lambda_{n}=1$ for $n \neq 0$. Physically, for $n \neq 0, V_{m, n}^{+}$is equivalent to $V_{m, n}^{-}$up to a rotation of $\pi / 2 n$ in momentum space. Different generalized PPs are mutually orthogonal: $\int d^{2} q V_{m, n}^{\sigma}(\vec{q}) V_{m^{\prime}, n^{\prime}}^{\sigma^{\prime}}(\vec{q})=\delta_{m, m^{\prime}} \delta_{n, n^{\prime}} \delta_{\sigma, \sigma^{\prime}}$, and any effective interaction can be expanded as

$$
\begin{aligned}
V_{\vec{q}} & =\sum_{\substack{m, n=0 \\
\sigma= \pm}}^{\infty} c_{m, n}^{\sigma} V_{m, n}^{\sigma}(\vec{q}), \\
c_{m, n}^{\sigma} & =\int d^{2} q V_{\vec{q}} V_{m, n}^{\sigma}(\vec{q}) .
\end{aligned}
$$

It is thus clear that we recover the Haldane PPs as special cases of $V_{m, n}^{+}$with $n=0$ (and $V_{m, 0}^{-}=0$ ), so we can write $V_{m, 0}^{+}=V_{m}, c_{m, 0}^{+}=c_{m}, c_{m, 0}^{-}=0$. For fermions, the shortest-range generalized PP is $V_{1, n}$; the quadrupolar contour profile of $V_{1,2}$ is clearly visible when compared against the isotropic $V_{1}$ Haldane PP (Fig. 1).
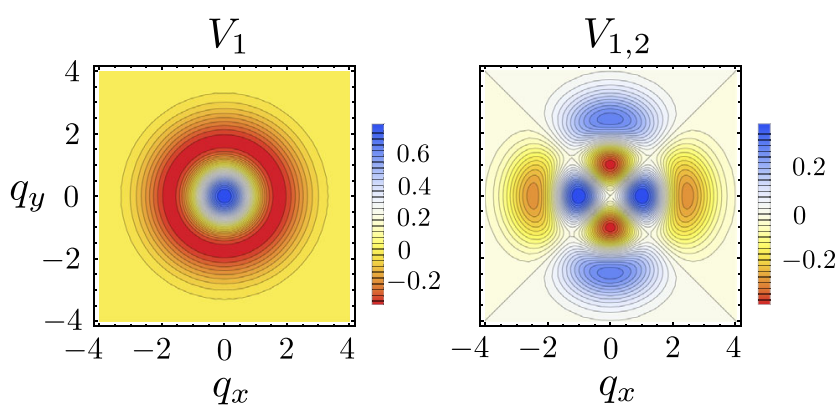

FIG. 1. Contours of the leading-order fermionic $V_{1}$ (isotropic) and $V_{1,2}^{+}$anisotropic pseudopotential. 
Intra-LL molecules.-To gain some intuition about the generalized PPs, it is instructive to study the spectrum of the simplest anisotropic PP, $V_{1,2}^{+}$, for small numbers of electrons on an infinite disk. From Eq. (2) it is clear that the ground state is a bound state with negative energy. The bound states can be understood as "molecular levels" of these particles, and the guiding-center density profile of such molecules in the center of mass (guiding-center) angular momentum $M=0$ sector are plotted in Fig. 2 .

Note that for anisotropic Hamiltonians the effect of the disk boundary cannot be removed for any finite system size. This means that $M$, in contrast to rotationally symmetric systems, is no longer a good quantum number for any eigenstate in the presence of the boundary. For bound states in a large disk, however, the particle density decays exponentially towards the boundary, so that the diagonalization within sub-Hilbert spaces of small $M$ is an excellent approximation. The guiding-center density plots in Fig. 2 thus represent very well the molecular structures of the few particle states in an infinite plane at $M=0$. For three electrons, the ground state reflects an interesting bonding structure between the particles. There is also a localized antibonding state at the highest energy, and the spectrum as a function of $M$ is symmetric about the zero energy. These properties are qualitatively the same for any small cluster, and are studied in detail elsewhere.

One unique feature of particles within a single LL (whose kinetic energy is quenched) is that the bound states exist even for purely repulsive interaction. For example, take two particles with interaction $V_{\vec{q}}=V_{1}+\lambda V_{1,2}^{+}$, The ground state of this system is a bound state regardless of
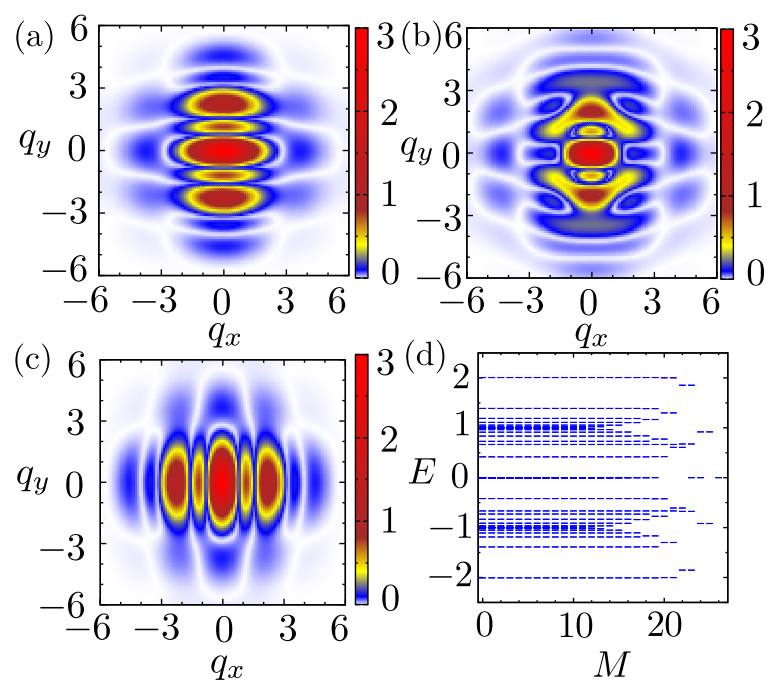

FIG. 2. The guiding-center density $\left\langle\Psi\left|\bar{\rho}_{\vec{q}}\right| \Psi\right\rangle[(\mathrm{a})-(\mathrm{c})]$ and energy spectrum (d) of a 3-electron molecule on the disk with 30 magnetic orbitals. The density is evaluated in the ground state (a), first-excited state (b), and highest-energy "antibonding" state (c). The states are obtained by exact diagonalization of $V_{\vec{q}}=V_{1,2}^{+}$ in the $M=0$ sector. how small $\lambda$ is. One can easily construct an effective interaction $V_{\vec{q}}$ consisting of $V_{0}, V_{1}, V_{2}$, and $V_{1,2}^{ \pm}$, such that $V_{\vec{q}}>0$ for all $\vec{q}$. Equation (1) has bound states even when the interaction is repulsive everywhere, and the properties of these bound states are qualitatively the same as those in Fig. 2, with the molecule size increasing as $\lambda$ decreases. This unique feature is a direct consequence of the quenched kinetic energy and particle statistics, and does not apply to free particles in two-dimensional space, where no bound states exist for repulsive interactions.

Physical applications.-We now provide two applications of the generalized PPs. First, we show that generalized PPs are a natural probe of the FQH nematic states [22,23]. Second, we demonstrate that anisotropic PPs emerge naturally in FCIs [26], where they can be comparable in magnitude to the isotropic PPs.

The FQH nematic $[22,23,27-30]$ is a phase with topological order and a charge gap, but at the same time spontaneously breaking rotational symmetry like a liquid crystal [31]. Recent interest in such phases is fuelled by experiment $[20,25]$, and effective field theories [22,23] that describe nematic states as the long-wavelength instability of the quadrupolar spin-2 excitation of the Laughlin state [11]. On the other hand, identifying nematic phases in microscopic models has proven more subtle. Recent work [24] suggested that a nematic phase might be realized in a model containing $V_{1}, V_{3}$, and $V_{5}$ Haldane PPs. However, the nematic phase was diagnosed by its response to changes in the sample geometry, which modifies all the interaction PPs at the same time, thus not conclusively ascertaining the quadrupolar character of the phase.

The generalized PPs can be used as a diagnostic of the nematic phase by measuring the response to small perturbations of the isotropic potential by $V_{m, n}$, while preserving the geometry of the sample. In Fig. 3(a) we plot the nematic response under adding or subtracting a small amount of $\delta V_{1,2} \sim 10^{-3}$. The nematic susceptibility plotted in Fig. 3(a) is defined as $\chi \equiv\left|\mathcal{N}\left(V_{m} ; \delta V_{1,2}\right)-\mathcal{N}\left(V_{m} ;-\delta V_{1,2}\right)\right| /$ $\left(2 \delta V_{1,2}\right)$, where the nematic order $\mathcal{N}$ is given by [24] $\mathcal{N} \equiv$ $\left(1 / N_{\Phi}\right) \sum_{\vec{q}} S_{\vec{q}}\left(\cos q_{x}-\cos q_{y}\right)$, in terms of the guidingcenter structure factor, $S_{\vec{q}}$ [2]. The data are for 8 electrons at filling $\nu=1 / 3\left(N_{\Phi}=24\right.$ flux quanta) on a square torus. Because of the gapless nature of the nematic phase, the response was averaged over the 50 lowest eigenstates. It is clear that the nematic response strongly peaks around $V_{3} \approx V_{5} \approx 0.6$; the location of the peak is in agreement with Ref. [24], while at the same time the finite-size effect is reduced.

Another advantage of the method presented here is that instead of $V_{1,2}$, we can controllably vary other PPs such as $V_{1,4}, V_{3,2}$, etc. Interestingly, the nematic susceptibility under $\delta V_{1,4}$ is strongly suppressed on the scale of Fig. 3(a). The response under $\delta V_{3,2}$ is qualitatively similar to $\delta V_{1,2}$, but smaller in magnitude. These results suggest 

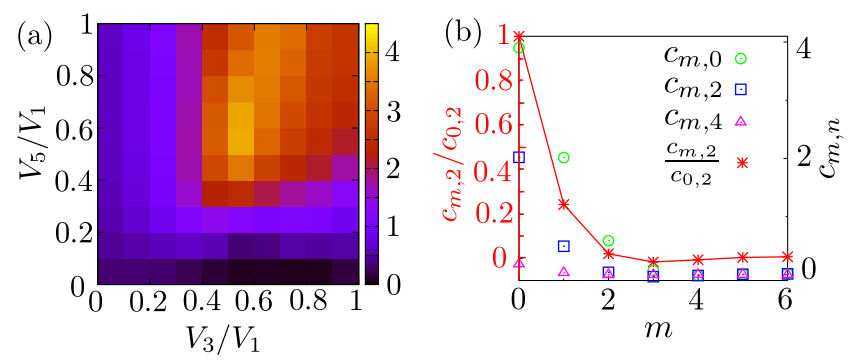

FIG. 3. (a) Nematic susceptibility $\chi$ in a model with isotropic $V_{1}=1, V_{3}$, and $V_{5}$ Haldane PPs. The results are averaged over 50 lowest states of a system of eight electrons on a square torus. The putative nematic phase [24] occurs around $V_{3} \approx V_{5} \approx 0.6$. (b) Anisotropic PPs in a FCI model. Left: the coefficients of anisotropic PPs for a quadrupole interaction $V_{q}=\cos q_{x}-\cos q_{y}$, whose isotropic PPs vanish. The first two PPs dominate. Right: PP magnitudes $c_{m, 0}, c_{m, 2}, c_{m, 4}$ for $V_{q}=\cos q_{x} . V_{m, 2}$ is of comparable magnitude as the isotropic $\mathrm{PP} V_{m}$, at least for $m=0$ and 1 .

the quadrupolar nature of the nematic phase, resulting from the instability of the long-wavelength part of the Laughlin magnetoroton mode [32]. This is also confirmed by the variational calculations using the magneroton wave functions [33], which show the neutral excitation going soft at small momenta in the nematic region of Fig. 3(a). It would be interesting to construct generalized nematic states whose primary instabilities occur in octupolar and higher order channels.

wAs a second application, we show that large anisotropic PPs naturally arise in FCIs [34-38]. Since interactions on a lattice are intrinsically directional, FCIs can possess anisotropic PPs that are even larger than the isotropic ones. The PP decomposition of a FCI requires a basis mapping between the single-body orbitals of the FCI and FQH systems [39-44]. We adopt the mapping introduced in Ref. [45], which maps a Chern number $C$ Chern band to a single LL with $C$-fold degeneracy [46] without introducing basis anisotropy $[39-41,44]$. For a clean realization of large PP FCIs, we choose a Chern insulator with almost uniform Berry curvature and Fubini-Study metric, so as to restrict rotational symmetry breaking to the interaction. Hence an interaction with predefined symmetry (i.e., $d$ wave) only leads to anisotropic PPs of that symmetry. Using the 3-band, Chern number 3 model [47], we plot in Fig. 3(b) its PP coefficients computed via Eq. (5) for nearest neighbor interactions of the (unprojected) form $V_{q}=\cos q_{x}$ or $V_{q}=\cos q_{x}-\cos q_{y}$. In the former case, $V_{m, 2}$ is of comparable magnitude with the isotropic $\operatorname{PP} V_{m, 0}$ (at least for $m=0$ and 1 ), while in the latter (quadrupole) case, the isotropic PPs cancel and we are left only with anisotropic PPs.

Metrics in generalized PPs.-The existence of a geometric degree of freedom for the dynamics within a single LL is the starting point of recent theoretical approaches to the FQH effect $[11,15-18,22,48]$. The incompressibility of $\mathrm{FQH}$ phases in general does not require rotational symmetry, but model wave functions (e.g., Laughlin [5], Moore-Read [9] states, etc.) are rotationally invariant, and the intrinsic metric in them appears as a "hidden" variational parameter. Quantum fluctuations of the metric determine the gap of the neutral excitations in the longwavelength limit $[11,33]$, which is crucial for the incompressibility of the topological fluids. Geometric aspects of the compressible quantum Hall phases can be more easily probed experimentally, especially in higher LLs [49,50].

While the importance of the metric in the many-body states has been recognized, the generalized PPs allow us to explicitly study the metric of the projected Hamiltonian of Eq. (1). It is straightforward to extend the generalized PPs above to the case of a general metric $g$. For convenience, we introduce a complex vector $\omega^{a}$ such that $\epsilon^{a b} \omega_{a}^{*} \omega_{b}=i$; then $g^{a b}=\omega^{a *} \omega^{b}+\omega^{a} \omega^{b *}$. Further, we define

$$
\mathbf{q}=\omega^{a} q_{a}, \quad|q|^{2}=g^{a b} q_{a} q_{b} .
$$

With the above redefinitions, the expression for the PPs, Eqs. (3) and (4), as well as the orthogonality relations, continues to hold. This means that in general, we can expand any interaction as

$$
V_{\vec{q}}=\sum_{m, n, \sigma} c_{n, m}^{g \sigma} V_{n, m}^{g \sigma}(\vec{q})
$$

More generally, because of the $S O(2,1)$ invariance of the integration measure in the Hamiltonian (1), we find that arbitrary two-body interactions are divided into equivalence classes where the members of the same family are interactions related to each other by a stretch and rotation of the guiding center metric. In other words, we can generate a family of interactions from the rhs of Eq. (7) that have the same $\left\{c_{n, m}^{g \sigma}\right\}$, but different PP metric $\eta$ in $V_{n, m}^{\eta \sigma}(\vec{q})$; all such interactions lead to the same spectrum upon substituting in Eq. (1). For certain $V_{\vec{q}}$ that are anisotropic in the lab frame, one can tune the metric in Eq. (7) to minimize the coefficients of the anisotropic PPs. More importantly, for gapped $\mathrm{FQH}$ fluids, we can truncate the expansion of the anisotropic interaction into the generalized PPs with appropriate metric. This would give new classes of anisotropic model Hamiltonians that could provide simpler description of topological phases and phase transitions where geometry plays an important role.

The technical details on explicitly defining the variational metric in the PPs are outlined in [47]. It can be shown that for gapped FQHE phases, the main effect of introducing particular anisotropy (i.e., by adding selected $V_{m, n}^{ \pm}$) is to change the emergent metric of the gapped ground state, without significantly reducing the incompressibility gap. Similarly, tilting the magnetic field for a quantum Hall sample with a finite thickness can be shown to introduce rather small anisotropy, even with a very large in-plane magnetic field [47].

Conclusions. - We have formulated a notion of generalized PPs that completely describe any two-body effective 
interactions within a single LL. This allows for a systematic way to quantitatively describe anisotropic $\mathrm{FQH}$ systems, such as tilted magnetic field systems, nematic states, and FCIs. The generalized PPs, possibly in combination with standard isotropic PPs, may give rise to new topological states with broken symmetry at finite filling factors. It would be interesting to study the dynamics of the few-particle molecular bound states for purely anisotropic PPs in the limit of vanishing filling factor, as well as the many-body phases at large anisotropy, which is ubiquitous in realistic FCI models.

This work was supported in part by Singapore Agency for Science, Technology and Research (A*STAR) Science and Engineering Research Council (SERC) "Complex Systems" Research Program Grant No. 1224504056, and the Department of Energy, Office of Basic Energy Sciences through Grant No. DE-SC0002140. Z.-X. H. was supported by National Natural Science Foundation of China Grants No. 1127403, No. 11674041, and No. 91630205 and Fundamental Research Funds for the Central Universities Grant No. CQDXWL-2014-Z006. Z.P. acknowledges support by EPSRC Grant No. EP/ P009409/1.

[1] D. C. Tsui, H. L. Stormer, and A. C. Gossard, Phys. Rev. Lett. 48, 1559 (1982).

[2] R. Prange and S. Girvin, The Quantum Hall Effect, Graduate Texts in Contemporary Physics (Springer-Verlag, Berlin, 1987).

[3] F. D. M. Haldane, Phys. Rev. Lett. 51, 605 (1983).

[4] F. D. M. Haldane, The Quantum Hall Effect (Springer, New York, 1990).

[5] R. B. Laughlin, Phys. Rev. Lett. 50, 1395 (1983).

[6] S. A. Trugman and S. Kivelson, Phys. Rev. B 31, 5280 (1985).

[7] S. H. Simon, E. H. Rezayi, and N. R. Cooper, Phys. Rev. B 75, 075318 (2007).

[8] C. H. Lee, Z. Papić, and R. Thomale, Phys. Rev. X 5, 041003 (2015).

[9] G. Moore and N. Read, Nucl. Phys. B360, 362 (1991).

[10] N. Read and E. Rezayi, Phys. Rev. B 59, 8084 (1999).

[11] F. D. M. Haldane, Phys. Rev. Lett. 107, 116801 (2011).

[12] T. Can, M. Laskin, and P. Wiegmann, Phys. Rev. Lett. 113, 046803 (2014).

[13] B. Bradlyn and N. Read, Phys. Rev. B 91, 125303 (2015).

[14] A. Gromov, G. Y. Cho, Y. You, A. G. Abanov, and E. Fradkin, Phys. Rev. Lett. 114, 016805 (2015).

[15] R.-Z. Qiu, F. D. M. Haldane, X. Wan, K. Yang, and S. Yi, Phys. Rev. B 85, 115308 (2012).

[16] B. Yang, Z. Papić, E. H. Rezayi, R. N. Bhatt, and F. D. M. Haldane, Phys. Rev. B 85, 165318 (2012).

[17] H. Wang, R. Narayanan, X. Wan, and F. Zhang, Phys. Rev. B 86, 035122 (2012).

[18] V. M. Apalkov and T. Chakraborty, Solid State Commun. 177, 128 (2014).

[19] Z. Papić, Phys. Rev. B 87, 245315 (2013).
[20] J. Xia, J. P. Eisenstein, L. N. Pfeiffer, and K. W. West, Nat. Phys. 7, 845 (2011).

[21] M. Mulligan, C. Nayak, and S. Kachru, Phys. Rev. B 82, 085102 (2010).

[22] J. Maciejko, B. Hsu, S. A. Kivelson, Y. J. Park, and S. L. Sondhi, Phys. Rev. B 88, 125137 (2013).

[23] Y. You, G. Y. Cho, and E. Fradkin, Phys. Rev. X 4, 041050 (2014).

[24] N. Regnault, J. Maciejko, S. A. Kivelson, and S. L. Sondhi, arXiv:1607.02178.

[25] N. Samkharadze, K. A. Schreiber, G. C. Gardner, M. J. Manfra, E. Fradkin, and G. A. Csathy, Nat. Phys. 12, 191 (2016).

[26] T. S. Jackson, G. Moller, and R. Roy, Nat. Commun. 6, 8629 EP (2015).

[27] L. Balents, Europhys. Lett. 33, 291 (1996).

[28] K. Musaelian and R. Joynt, J. Phys. Condens. Matter 8, L105 (1996).

[29] M. Mulligan, C. Nayak, and S. Kachru, Phys. Rev. B 82, 085102 (2010).

[30] M. Mulligan, C. Nayak, and S. Kachru, Phys. Rev. B 84, 195124 (2011).

[31] P. G. de Gennes and J. Prost, The Physics of Liquid Crystals, International Series of Monographs on Physics (Clarendon Press, New York, 1995).

[32] S. M. Girvin, A. H. MacDonald, and P. M. Platzman, Phys. Rev. Lett. 54, 581 (1985).

[33] B. Yang, Z.-X. Hu, and Z. Papić, and F. D. M. Haldane, Phys. Rev. Lett. 108, 256807 (2012).

[34] D. N. Sheng, Z.-C. Gu, K. Sun, and L. Sheng, Nat. Commun. 2, 389 (2011).

[35] K. Sun, Z. Gu, H. Katsura, and S. Das Sarma, Phys. Rev. Lett. 106, 236803 (2011).

[36] E. Tang, J.-W. Mei, and X.-G. Wen, Phys. Rev. Lett. 106, 236802 (2011).

[37] T. Neupert, L. Santos, C. Chamon, and C. Mudry, Phys. Rev. Lett. 106, 236804 (2011).

[38] N. Y. Yao, A. V. Gorshkov, C. R. Laumann, A. M. Läuchli, J. Ye, and M. D. Lukin, Phys. Rev. Lett. 110, 185302 (2013).

[39] X.-L. Qi, Phys. Rev. Lett. 107, 126803 (2011).

[40] C.-M. Jian and X.-L. Qi, Phys. Rev. B 88, 165134 (2013).

[41] C. H. Lee, R. Thomale, and X.-L. Qi, Phys. Rev. B 88, 035101 (2013).

[42] Y.-L. Wu, B. A. Bernevig, and N. Regnault, Phys. Rev. B 85, 075116 (2012).

[43] Y.-L. Wu, N. Regnault, and B. A. Bernevig, Phys. Rev. Lett. 110, 106802 (2013).

[44] C. H. Lee and X.-L. Qi, Phys. Rev. B 90, 085103 (2014).

[45] M. Claassen, C. H. Lee, R. Thomale, X.-L. Qi, and T. P. Devereaux, Phys. Rev. Lett. 114, 236802 (2015).

[46] It thus avoids the complications of inter-LL mixings introduced by alternative mapping schemes (but see Ref. [43]).

[47] See Supplemental Material at http://link.aps.org/ supplemental/10.1103/PhysRevLett.118.146403 for more technical details.

[48] F. D. M. Haldane, arXiv:0906.1854.

[49] M. P. Lilly, K. B. Cooper, J. P. Eisenstein, L. N. Pfeiffer, and K. W. West, Phys. Rev. Lett. 82, 394 (1999).

[50] R. Du, D. Tsui, H. Stormer, L. Pfeiffer, K. Baldwin, and K. West, Solid State Commun. 109, 389 (1999). 